\title{
Long Slot mmWave Low-SLL Periodic-Modulated Leaky-Wave Antenna Based on Empty SIW
}

\author{
Ali Araghi, Graduate Student Member, IEEE, Mohsen Khalily, Senior Member, IEEE, \\ Pei Xiao, Senior Member, IEEE, Rahim Tafazolli, Senior Member, IEEE, and David R. Jackson, Fellow, IEEE
}

\begin{abstract}
In this paper, empty substrate integrated waveguides (ESIW) technology is applied to design long slot leaky-wave antennas (LWAs). First, a uniform-aperture structure is presented and its limitations on forming the beam are studied. Then, a sinusoidal curve is employed to modify the geometry of guidedwave structure which divides the slot into a number of segments, making a periodic aperture. After that, a method is proposed to regulate the guided waves inside the ESIW. To this end, a modulation function is derived to simultaneously determine the local amplitude and segment length of the physical sinusoidal curve at each individual points on the structure. This results in manipulating the phase constant $(\beta)$ and leakage rate $(\alpha)$ across the aperture which ultimately controls both the tilt angle and side-lobe-level (SLL) of the constructed beam. The slot is placed on the centerline of the broad wall of the ESIW in order to reduce the cross polarization. The structure is designed to operate at $35 \mathbf{G H z}$ with $\mathbf{S L L}=-30 \mathbf{d B}$ and a backward tilt angle of $\theta_{m}=-20$ deg. Finally, the proposed LWA is simulated and a fabricated design is measured. A good agreement is observed between the theoretical, simulated, and measured performance of the antenna.
\end{abstract}

Index Terms-Leaky-wave antenna (LWA), modulated structures, empty substrate integrated waveguides (ESIW), periodic LWA.

\section{INTRODUCTION}

$\mathbf{L}$ EAKY-Wave antennas (LWAs) are realized by regulating the coupled power per unit-length from an electromagnetic (EM) aperture on a guided-wave structure to the free space [1]. They exhibit many unique advantages such as relatively high gain, ease of fabrication, large bandwidth, and inherent beam-scanning capability with a simple feed network. These features make them a promising candidate to be applied in the millimeter wave (mmWave) band and terahertz regimes [2], [3].

The aperture can be formed by applying purposeful modifications on the geometry of the structure in order to make the guided waves leak to the open region. This leakage must be managed in a way such that the power flow of the propagating waves is exponentially increasing in the air region as they

This work was supported by 5GIC \& 6GIC, Institute for Communication Systems (ICS), University of Surrey, Guildford, UK, and the EPSRC under the grant number EP/P03456X/1.

Ali Araghi, Mohsen Khalily, Pei Xiao, and Rahim Tafazolli are with the 5GIC \& 6GIC, Institute for Communication Systems (ICS), University of Surrey, Guildford, GU2 7XH, UK, (e-mail: \{a.araghi, m.khalily, p.xiao, r.tafazolli\}@surrey.ac.uk).

David R. Jackson is with the Department of Electrical and Computer Engineering, University of Houston, Houston, TX 77204-4005, USA (e-mail: djackson@uh.edu). get distanced from the structure in a direction normal to the structure's plane. Under this circumstance, the leaky-waves are often described as being "improper" or "nonspectral" [4] and the structure in hand is the so-called LWA.

The aperture can be shaped in a variety of forms such as a series of transversal slots on a waveguide's wall [5], a set of sub-wavelength metallic scatterers printed on a substrate [6], or a long slot on a wall of guided-wave structure that cuts the surface current lines [7]. Among all various types of long-slot LWA, the longitudinal straight case has the most straightforward configuration judging from the manufacturing perspectives [8]. However, a uniform straight slot results in high side-lobe-level (SLL) which is inappropriate for many applications.

LWAs are characterised by the leakage rate and phase constant across the aperture. Independent control over these two parameters is necessary to allow beam shaping. In order to control the SLL, the leakage rate should be manipulated while phase constant specifies the pointing angle.

In the mmWave band, waveguide devices are typically preferred to the microstrip ones from the "power loss" and "power handling" points of view. However, the fabrication process of these devices could be difficult, especially for designs requiring non-conventional waveguide bodies. Investigations on substrate integrated waveguides (SIWs) show that SIW properties are transitional between microstrip and dielectricfilled waveguide properties [9], [10]. Prominent advantages of SIW are low cost, light weight, and easy integration with planar circuits. As a result, a number of long-slot LWA designs have been proposed based on SIW technology. For example Cheng et al. [8] propose a structure in which the SIW's sidewalls are meandered to control the rate of the leakage and obtain a low-SLL beam in the mmWave band. Meandering the sidewalls enables them to put the slot in the middle providing a well cross-polar cancellation; however, there is no particular control on the direction of constructed beam. A pair of long slots are etched symmetrically on the top broadwall of an SIW working in the $\mathrm{TE}_{20}$ mode [11]. With the aid of field distribution pattern of this mode on both sides of slots, and by controlling the aperture amplitude illumination, both SLL and cross-pol are managed to meet a predefined criteria, again with no specific mechanism of adjusting the pointing angle. Using transverse equivalent technique, a low SLL beam with low cross-pol component is achieved in the X-band by a ridged SIW [12]. But the structure requires at least two substrate layers to make the geometry of ridges. With the same concept, an X-band sinusoidal ridged SIW long slot LWA is presented 
in [13]. This design was inspired by a wiggly-ridged long slot LWA using a conventional waveguide [14].

Despite the many advantages of SIWs, they may fail to be properly employed in LWAs whenever the dielectric loss of the substrate is not negligible. In this situation, the leakage rate may deviate from what exactly it meant to be, which would ultimately deteriorate the constructed beam. This issue is more prominent in the mmWave band where the dielectric loss of substrates is relatively high. Hence, it is generally preferred to use substrate materials with extremely low tangent loss or ceramics. As mentioned earlier, one advantage of using SIWs is their possible integration with the RF circuits on a same board. These RF components may require some specific dielectric properties for particular reasons. In many cases, it can be imagined that the RF components may need an especial substrate where the performance of the SIW LWA would not be optimal with that substrate. For example, it is generally preferable to employ substrates with higher dielectric constant to make the RF components compact. The loss tangent of these substrates is usually high which is not a concern for a small size RF component. But it may alter the constructed beam of the LWA as it is explained.

Therefore, in an integrated board, the designers would ideally need a technique to make the SIW LWA's substrate independent from the rest of the board, but at the same time, the integrity of board remains intact.

Recently by removing the dielectric body enclosed by lateral metallized holes of the traditional SIWs, a new guided wave structure is introduced which is called empty SIW (ESIW) [15]-[17]. In the ESIWs, the EM-waves are confined by the lower, lateral, and upper metallized walls while travelling throughout an air-filled material; therefore the dielectric loss will almost be completely eliminated. As the EMcharacteristics of the substrate do not play an important role on the guided-wave's travelling path, it is concluded that ESIW can be the technique for making the radiating aperture independent from other components on the board. The RFcomponents can benefit from a specific substrate as required without being worried about the antenna side.

To the best of our knowledge, the ESIW has been mostly applied to design RF-components so far and its application in the antenna domain is limited to an H-plane horn [18] and few designs on resonating arrays [19]-[21].

In this paper, we propose an original mechanism to control the leakage constant (and SLL) of a long-slot LWA by introducing a periodic-modulated structure based on the ESIW for the first time. The proposed approach can successfully control the direction of beam while placing the slot in the middle leading to a low cross-pol radiation pattern. The structure is designed to operate at around $f=35 \mathrm{GHz}$.

\section{BRIEF OVERVIEW ON LWAS}

A guided wave structure with a 1-D aperture along the $x$-axis has a propagation constant of $k_{x}=\beta_{x}-j \alpha_{x}$ where $\beta_{x}$ and $\alpha_{x}$ are phase and loss constants across $x$ respectively. The air-filled closed-structures support fast-waves for all propagating modes with $\left|\beta_{x}\right|<k_{0}$, with $k_{0}$ being the free space wave number. However, dielectric-filled and dielectric slabs contain slow-waves with $\left|\beta_{x}\right|>k_{0}$ [1]. An aperture on a fast-wave structure may result in an LWA, but slow-wave structures in their basic form cannot radiate toward any direction other than the (near) endfire or backfire [22].

EM-apertures for LWAs are typically categorized as "uniform", "quasi-uniform", and "periodic" according to their distribution [23], [24]. The aperture's physical shape of the uniform structure does not change along the direction of guided waves; it can also gradually vary to control the leakage constant, but still fall into the uniform category [4].

The angle of constructed beam for a uniform-aperture LWA is determined by the following well-known equation:

$$
\sin \theta_{m} \approx \frac{\beta_{x}}{k_{0}} .
$$

The periodic structures consist of a geometry repeated locally and can be analyzed mainly based on the theory of space harmonics [25]. This physical repetition provides a periodicity in the spatial domain, which is expandable to Fourier series. The spatial Fourier expansion in this regard is known as Floquet spatial harmonic expansion [22]. Infinite number of space harmonics will be shaped with the propagation constant of

$$
k_{x n}=k_{x 0}+\frac{2 \pi n}{d}, n=0, \pm 1, \pm 2, \ldots
$$

where $d$ is the repetition period and $n$ is the harmonic index. The corresponding propagation constant of outward pointing wavenumber vector at the field-emanating interface is $k_{z n}=\sqrt{k_{0}^{2}-k_{x n}^{2}}$. The radiation condition is met for every $n$th harmonic located in the fast-wave region. Usually the fundamental mode $(n=0)$ is slow with $k_{x 0}>k_{0}$ and $n=-1$ spatial harmonic will meet the radiation condition with $-k_{0}<\beta_{-1}=\beta_{x}-2 \pi / d<k_{0}$. By considering Eq. (1) and Eq. (2), the direction of beam can be obtained by $\theta_{m} \approx \sin ^{-1}\left(\beta_{-1} / k_{0}\right)$. Consequently a periodic LWA can support both forward and backward beams while uniform LWAs lead to forward beam only (sign of $\beta_{x}$ and $k_{0}$ would be the same in Eq. (1)).

\section{UNIFORM-APERTURE LONG-SLOT LWA BASED ON ESIW: DESIGN, RESULT AND Discussions}

\section{A. Substrate Selection}

As explained in Section I, ESIW facilitates the antenna integration with RF-components on a common single-layer substrate. In order to demonstrate this notion, the substrate is chosen to be Rogers RO4360G2 with $\epsilon_{r}=6.4$ and high tangent loss of $\tan \delta=0.0038$. This substrate is typically used in RF-circuit boards [26], while its laminate processing is not complicated and is similar to that of FR-4 which is automated assembly compatible.

In an LWA, the overall attenuation constant that the guided waves experience is

$$
\alpha=\alpha_{l}+\alpha_{d}+\alpha_{c},
$$


where $\alpha_{l}, \alpha_{d}$, and $\alpha_{c}$ are the leakage rate, dielectric loss, and conduction (ohmic) loss respectively.

We can assume $\alpha_{d} \approx 0$ utilizing the ESIW, but $\alpha_{c}$ still needs to be considered, especially in the mmWave band.

Consider an SIW structure with substrate thickness of $h$ and equivalent width of $w_{g}$. As $h$ is relatively small in comparison with $w_{g}$, the propagation mode will be $\mathrm{TE}_{m 0}$. For the dominant mode of $\mathrm{TE}_{10}$, the conduction loss at the operating frequency of $f$ and cut-off frequency of $f_{c}$ is defined as below [27]:

$$
\left(\alpha_{c}\right)_{10}=\frac{R_{s}}{\eta h} \frac{1+\frac{2 h}{w_{g}}\left(\frac{f_{c}}{f}\right)^{2}}{\sqrt{1-\left(\frac{f_{c}}{f}\right)^{2}}}(\mathrm{~Np} / \mathrm{m}),
$$

where $R_{s}=\sqrt{\omega \mu_{0} / 2 \sigma}, \eta=\sqrt{\mu_{0} / \epsilon_{0} \epsilon_{r}}$, and $\sigma=5.7 \times$ $10^{7} \mathrm{~S} / \mathrm{m}$ (the conductivity of copper). Eq. (4) shows a higher value of $h$ results in lower $\alpha_{c}$. Therefore, to decrease the conductive loss, the substrate should be as thick as possible.

\section{B. Antenna Configuration and Performance}

The detailed design procedure of the back-to-back ESIW transmission line is presented in [15]. By adding a long slot on the upper wall of the ESIW, an LWA can be constructed as indicated in Fig. 1. The slot should be located with an offset $\left(o_{s}\right)$ from the middle of upper wall so that the surface current lines can be cut, hence the radiation would occur.

The first step is to find the value of $w_{g}$ in Fig. 1. In this design, the operating frequency is set to be at $f=35 \mathrm{GHz}$. As a rule of thumb, the operating frequency of any waveguidebase structure with the cut-off frequency of $f_{c}$ should be $1.5 f_{c}$. The bandwidth is defined between $1.25 f_{c}$ to $1.89 f_{c}$ [9] to guarantee enough distance between the operating mode and the next higher order mode. As $f=35 \mathrm{GHz}$, thus $f_{c}=$ $23.33 \mathrm{GHz}$, then by considering that $\left(f_{c}\right)_{10}=\frac{1}{2 \sqrt{\mu_{0} \varepsilon_{0}}} \sqrt{\frac{1}{w_{g}^{2}}}$ for the dominant $\mathrm{TE}_{10}$ mode, it can be calculated that $w_{g}=$ $6.425 \mathrm{~mm}$.

Among the standard dimensions of RO4360G2 laminate, the thickest one has the thickness of $h=1.524 \mathrm{~mm}$ which is selected to meet the aforementioned considerations on $\alpha_{c}$.

Following the method presented in [15], [16], the transition layout from microstrip to ESIW is designed with the parameters of $w_{m s}=1.07 \mathrm{~mm}, w t_{m s}=3 \mathrm{~mm}, w t_{i}=1.489 \mathrm{~mm}$, $l_{t}=2.14 \mathrm{~mm}, w_{i r}=5.3 \mathrm{~mm}$, and $d_{f}=1 \mathrm{~mm}$. The aperture parameters are selected as $l_{s l}=90 \mathrm{~mm}, l_{g}=100 \mathrm{~mm}$, $l_{\text {sub }}=122 \mathrm{~mm}, w_{\text {sub }}=20 \mathrm{~mm}$, and $w_{s}=0.3 \mathrm{~mm}$.

The proposed LWA is simulated by the CST-MWS and its EM performance for three values of $o_{s}=\{0.5,1,1.5\} \mathrm{mm}$, is reported in Fig. 2.

The simulated $\mathrm{S}_{11}$ shows that the structure is matched well through the entire band of study. As a result of slot's presence, a considerable amount of energy will leak to the open region, which can be interpreted from the $S_{21}$. At the operating frequency of $f=35 \mathrm{GHz},\left(\beta_{x}\right)_{10}=k_{0} \sqrt{1-\left(\frac{f_{c}}{f}\right)^{2}}=$ $k_{0} \times 0.7454$ and by considering Eq. (1), $\theta_{m}=48.19 \mathrm{deg}$. This is well aligned with the simulated results for the case

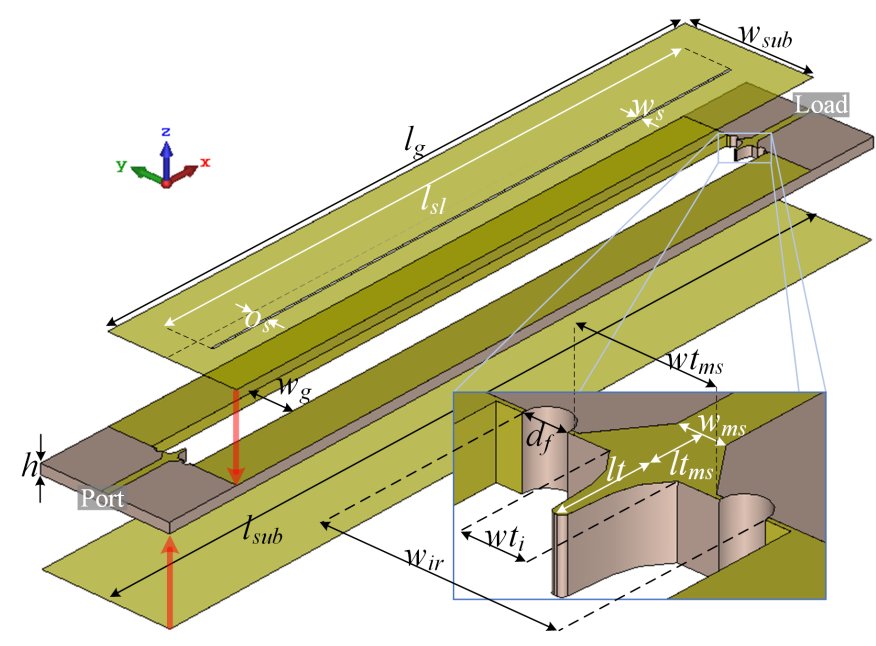

Fig. 1. The long slot LWA on ESIW.

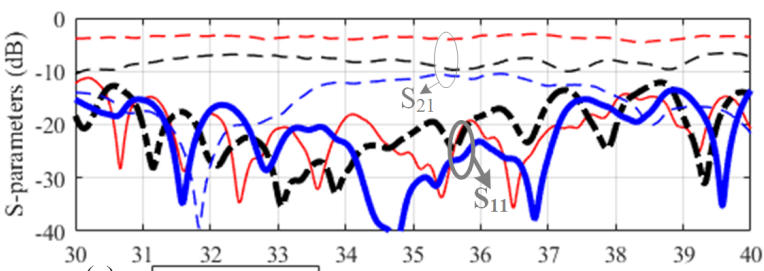

(a) $={ }^{\circ}=0.5 \mathrm{~mm}$ Frequency $(\mathrm{GHz})$

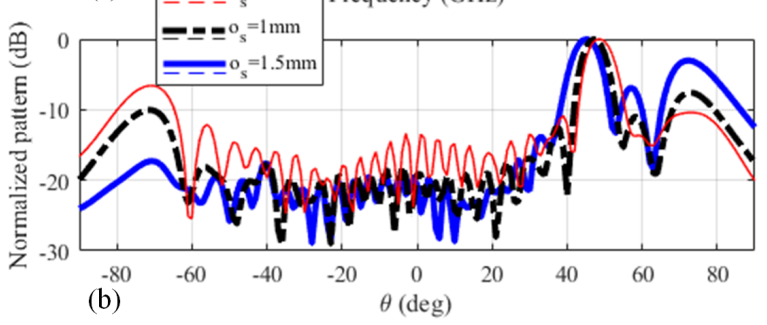

Fig. 2. The designed long slot LWA simulation performance (a) S-parameters and (b) normalized H-plane radiation pattern at $f=35 \mathrm{GHz}$.

$o_{s}=0.5 \mathrm{~mm}$. In this specific case, the comparably smaller value of offset will disturb the guided waves in a minor sense. Therefore the actual $\beta_{x}$ is almost equivalent to the theoretical value and this is why the simulated beam angle is better matched to the estimated one in this special case, comparing to the other cases.

Once the dimensions of guided-wave structure is set, there will be a subtle control on $\beta_{x}$ and subsequently the direction of the beam at a specific frequency. Furthermore, the uniform aperture is accompanied by the forward beam only. More importantly, the constructed pattern suffers from high SLL as shown in Fig. 2.

Now a question naturally arises: is there any particular approach to cope with all these restrictions?

\section{Periodic-Modulated Long-Slot LWA Design BASED ON ESIW}

In this section, by introducing a novel method, a long slot LWA on ESIW is presented which is capable of controlling 
both tilt angle and SLL of the radiation pattern simultaneously with a low cross-pol level. By applying a sinusoidally modulated guided-wave structure (SMGS), a periodic structure is obtained that can generate the spatial harmonics. In the rest of this section, we describe the design principles and details of the proposed method.

\section{A. Operation Principle of the Long-Slot LWA on an SMGS Based on ESIW Technology}

For a long-slot LWA, when the lateral-walls of the ESIW are curved with respect to the slot's orientation, the electric field distribution on both sides of the slot will become asymmetric and the radiation would then be possible. If this curve follows a sinusoidal pattern with period of $d$, the guided waves will become slow and the straight long slot will cut the surface currents periodically. By adjusting the value of $d$, the $n=-1$ spatial harmonic can meet the radiation condition and leakage would consequently occur (see Eq. (2)).

Based on the parameters specified in Fig. 1, the schematic view of the proposed sinusoidal guided-wave structure is presented in Fig. 3. For the ease of presentation, the effect of parameter $d$ is characterized by $S L$ as the length of each segment. The amplitude of the sinusoidal curve is denoted by $A_{m}$. The long slot is placed on the upper metallized wall of the ESIW. Due to the curved path of the guided waves, the slot can be placed in the middle, which can ultimately decrease the cross-pol level. As seen by the slot, there is a $\pi$ phase reversal in every $p$ distance (see Fig. 3). The effective axial phase velocity of the $n$th spatial harmonic is:

$$
\nu_{p h, n}=\frac{\omega}{\frac{\Delta \phi_{x, n}}{p}},
$$

where $\omega$ is the angular frequency of the wave $(\mathrm{rad} / \mathrm{s})$ and $\Delta \phi_{x, n}$ is the phase difference of the $n$th spatial harmonic's axial electric field between slot-crossings of the guided-wave structure.

$$
\beta_{x, n}=\frac{\Delta \phi_{x, n}}{p},
$$

is the effective axial wavenumber for the $n$th spatial harmonic.

For a structure with smooth variations of lateral walls, the $n$th axial electric field's phase shift between slot-crossings is expressed as:

$$
\Delta \phi_{x, n}=\left[\left(\frac{\omega}{\nu_{x, \text { wave }}}\right) p+\pi\right]+2 n \pi,
$$

where the term $\pi$ is for apparent phase reversal, $2 n \pi$ takes the space harmonics into account, and $\nu_{x, \text { wave }}$ is the axial phase velocity of the wave which can be calculated by:

$$
\nu_{x, \text { wave }}=\frac{p}{L_{c}} \frac{1}{\sqrt{\mu_{0} \epsilon_{0}} \sqrt{1-\left(\frac{f_{c}}{f}\right)^{2}}},
$$

with $L_{c}$ representing the length of each curved segment stated in Fig. 3.

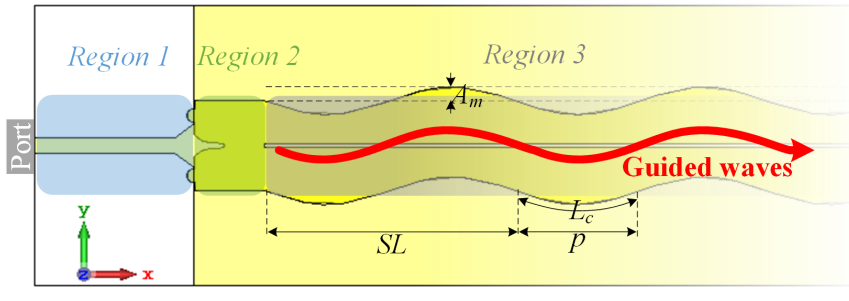

Fig. 3. The sinusoidal guided-wave structure with a long-slot on the upper broad wall of the ESIW: model for investigating the effect of physical geometry on the leakage rate and beam angle.

Considering Eqs. (6), (7), and (8), the effective wavenumber along $x$ for $A_{m} \neq 0$ can be approximately expressed by the following closed-form equation:

$$
\beta_{x, n}=k_{0}\left(\frac{L_{c}}{p}\right) \sqrt{1-\left(\frac{f_{c}}{f}\right)^{2}}+\frac{(2 n+1) \pi}{p} .
$$

Note that the value of $S L$ has a direct impact on the path that the guided waves are going to pass through. If the $S L$ is set proportionally small in comparison with the guided-wave's wavelength, the geometrical properties of the structure would then vary too rapidly to the extent that the guided waves cannot be formed. The value of $S L$ must be defined in a way that the guided waves experience gradual variations. It is necessary to consider this issue for the amplitude $A_{m}$ as well, which cannot be too large so that the unity of slot would be broken; abrupt variation of $A_{m}$ can also block the guided waves. In summary, the continuity of guided waves across the structure should be sustained so that the whole aperture can be illuminated properly.

\section{B. Overview on the Proposed Modulation Procedure}

The overall modulation process is illustrated in Fig. 4 and summarized hereinafter. The procedure is split into four steps: the first step is to define the aperture length, desired tilt angle, and the required SLL as the three input parameters of the whole process. In the second step, based on the aperture length and the defined tilt angle, the number of segments $(N)$ and their corresponding length $(S L)$ are determined (Section IV-C). Furthermore, the required distribution of $\alpha_{l}$ across the aperture must be defined with respect to the desired SLL. It will be shown in Section IV-D that the amplitude $A_{m}$ (see Fig. 3) has a direct impact on the $\alpha_{l}$. Hence, for a known distribution of $\alpha_{l}, A_{m}$ distribution would be clarified as well. For our specific LWA, it will be shown that $N$ and $A_{m}$ are two interrelated functions. Therefore, as the third step, for an arbitrary position of $x$ across the aperture, $A_{m}(x)$ and $N\left(A_{m}(x)\right)$ will be derived in Section IV-E, followed by calculating the modulation function $\mathcal{M}$. This obtained modulation function will characterize the physical geometry of the supporting SMGS. In Section IV-F, the desired LWA on the defined SMGS will be introduced which is the forth and final step of our proposed procedure. 


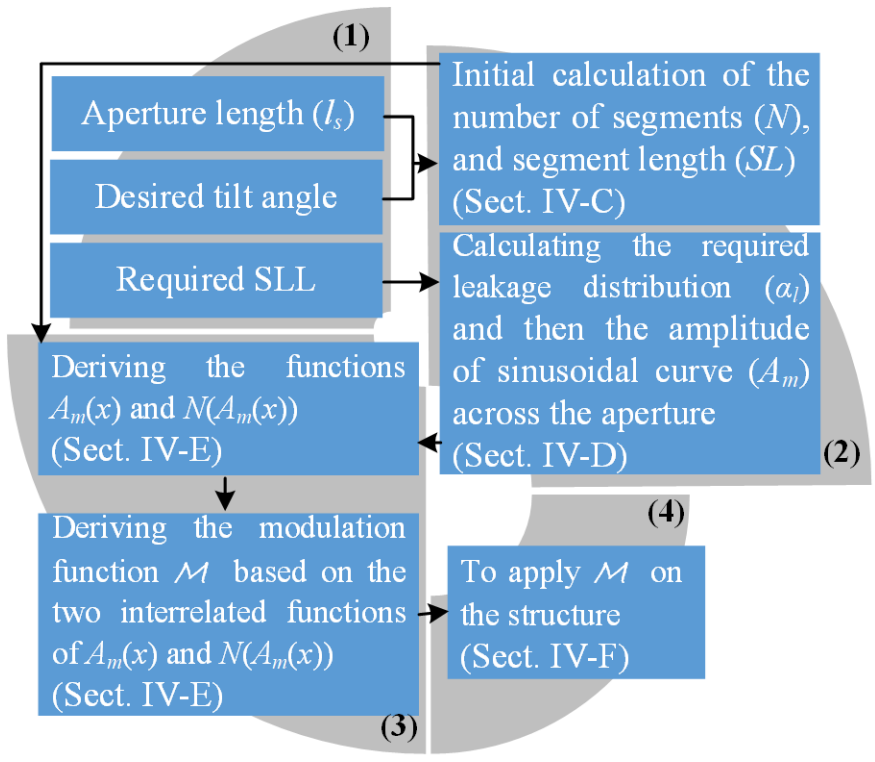

Fig. 4. Block diagram of the overall proposed modulation process.

\section{Relation Between Leakage Characteristics and Geometri- cal Parameters}

In the proposed structure shown by Fig. 3, a portion of wave's energy is cut by the long-slot. This portion would be changed as $A_{m}$ changes. Therefore, as long as the matching condition is satisfied, variations of $A_{m}$ would make the leakage rate vary. On the other hand, any alteration in $S L$ would change the number of occasions where the guided waves cross the slot. This would mostly alter the phase constant of the guided waves and consequently the tilt angle of the constructed beam (see Eq. (1)).

As $\alpha_{d}$ and $\alpha_{c}$ are negligible in the proposed structure, it is possible to increase the slot's length and have a larger aperture. The longer slot will typically result in higher gain, but more importantly, the higher number of wavelengths that can be formed in a longer aperture brings a flexibility to regulate the leakage rate and the phase constant in a more accurate fashion. Therefore, the slot length is set to be $l_{s}=190 \mathrm{~mm}$, which contains more than 16 wavelengths of the guided waves along the slot at $f=35 \mathrm{GHz}$. As we need to study and analyze several parameters across the slot's length hereinafter, we may require to point to specific regions (spots) on the slot. For simplicity, instead of defining so many indices, let us use the notation $l_{s}$ to point to the total length of slot (i.e. $190 \mathrm{~mm}$ ), and when referring to a specific region (spot), we denote it by $l_{s}=$ region (spot) throughout the text.

The relation between $N$ and the tilt angle $\theta_{m}$ is derived by running series of simulations in the CST-MWS for different values of $N$ and $A_{m}$ with $w_{s}=0.3 \mathrm{~mm}$.

First, for the fixed value of $A_{m}=1 \mathrm{~mm}, N$ varies from 10 to 30 . The corresponding $\mathrm{H}$-plane normalized radiation pattern is presented in Fig. 5 (a) for some specific values of $N$ among the range of study (to make the illustrated material easier to read). The result states that for $N=10 \sim 30, \theta_{m}=+16 \sim$ $-43 \mathrm{deg}$ (It is notable that the tilt angle can be in both forward and backward directions as the proposed structure is periodic).
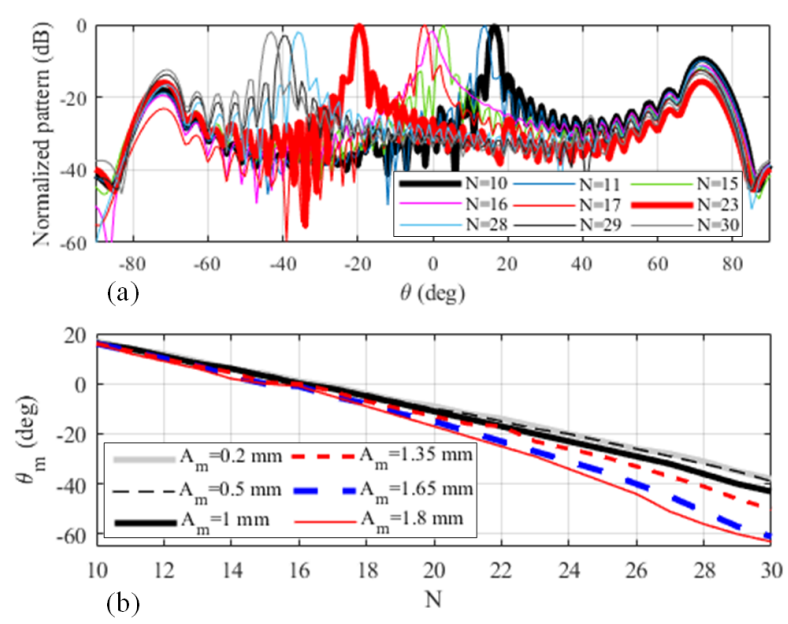

Fig. 5. The relation between the number of segments $(N)$ and the constructed beam angle $\left(\theta_{m}\right)$, (a) the H-plane radiation patterns for constant value of $A_{m}=1 \mathrm{~mm}$ and different values of $N$, (b) the $\theta_{m}$ vs. $N$ curve for different values of $A_{m}$. For all studied cases, $w_{s}=0.3 \mathrm{~mm}$.

In other words, depending on the value of $N$, it is possible to direct the beam toward any angle within this range.

In addition to the studied case of $A_{m}=1 \mathrm{~mm}$, the discussed series of simulations are repeated for the cases $A_{m}=$ $\{0.2,0.5,1.35,1.65,1.8\} \mathrm{mm}$ as illustrated in Fig. 5 (b). Given the curve of $A_{m}=1 \mathrm{~mm}$ as the reference, other curves indicate how much the tilt angles deviate. More explanations on this result will be provided later in this section.

Referring to Fig. 5 (a), it is instructive to point out that $N=16$ is accompanied by broadside radiation $\left(\theta_{m}=0 \mathrm{deg}\right)$ and therefore the stop-band effect [25]. This is the reason why the radiation pattern is distorted in this specific case. Furthermore, for all values of $N$, two comparably strong lobes occur at around $\theta_{m}= \pm 70 \mathrm{deg}$. These lobes also occur in the uniform case as can be seen from Fig. 2 (b). The source of these lobes are the microstrip transmission lines at both ends of the structure, i.e. Region 1 of Fig. 3. For the ESIW, the surface waves on the microstrip part are smoothly transformed into the guided waves in the air-filled part (from Region 1 to Region 2). Due to this transformation, the field distribution is intensified in both microstrip-line regions. The region that is containing the excitation port will have the backward lobe while forward lobe occur at the load side.

In this design, $\theta_{m}=-20 \mathrm{deg}$ is chosen for the beam pointing angle, then the proportionate value is $N=23$ according to the reference case of $A_{m}=1 \mathrm{~mm}$ in Fig. 5 (b).

In order to control the SLL, the parameter $A_{m}$ must be managed in a way that different parts of the aperture lead to a controlled amount of the leakage rate. To do so, the relation between $A_{m}$ and $\alpha_{l}$ needs to be identified in advance.

For a lossless 2-port transmission line, $\left|S_{11}\right|^{2}+\left|S_{21}\right|^{2}=1$, while for a lossy one with the length of $L$, this expression can be modified as follows:

$$
\left|\mathrm{S}_{11}\right|^{2}+\left|\mathrm{S}_{21}\right|^{2}=\frac{P(L)}{P(0)}=e^{-2 \alpha L},
$$


where $P(0)$ is the power at source and $P(L)$ is the delivered power to the load with $\alpha$ specifying by Eq. (3).

It is discussed that for ESIWs, $\alpha \approx \alpha_{l}$ so that Eq. (10) can be used to derive the dependency of $\alpha_{l}$ on different values of $A_{m}$ by studying the S-parameters in the corresponding fullwave simulations.

For three different values of $w_{s}=\{0.15,0.3,0.45\} \mathrm{mm}$, and constant value of $N=23$, the $A_{m}$ is altered from $0.01 \mathrm{~mm}$ to $2 \mathrm{~mm}$ and the structures are simulated. Concerning Eq. (1), $\sin \theta_{m}=\frac{\beta_{-1}}{k_{0}}$ which means that reading the resultant value of $\theta_{m}$ in each simulation enables us to find out the corresponding value of $\beta_{-1} / k_{0}{ }^{1}$. By using Eq. (10) in each simulation, $\alpha_{l}$ is calculated as shown in Fig. 6 alongside the curve of $\beta_{-1} / k_{0}$. Ideally, for all values of $A_{m}$, the aperture should radiate toward the same direction. However, the $\beta_{-1} / k_{0}$ curves reveal that for the studied structure, $\alpha_{l}$ and $\beta_{x}$ are not independent parameters; this is also evident from Fig. 5 (b).

As can be observed from the Fig. 6, a higher slot's width results in a smaller range of $\alpha_{l}$ 's variation. For the cases $w_{s}=0.15 \mathrm{~mm}$ and $0.3 \mathrm{~mm}$, the curves are terminated at $A_{m}=1.7 \mathrm{~mm}$ and $1.8 \mathrm{~mm}$ respectively as further increment in $A_{m}$ makes the structure mismatched. This is the reason why the upper limit of $A_{m}$ in Fig. 5 (b) is set to $1.8 \mathrm{~mm}$; while the lower limit of $A_{m}=0.2 \mathrm{~mm}$ in this figure comes from the result presented in Fig. 6. According to this figure, the variation of $\alpha_{l}$ for $A_{m}<0.2 \mathrm{~mm}$ is negligible and therefore $A_{m}=0.2 \mathrm{~mm}$ is chosen as the start point of study in Fig. 5 (b).

As shown in Fig. 6, $\alpha_{l}$ raises slightly when $A_{m}$ is swept from $0.2 \mathrm{~mm}$ to $0.5 \mathrm{~mm}$. For relatively higher values of $A_{m}$, the variations of $\beta_{-1} / k_{0}$ is steeper which lead to sharper variation of beam angle.

$A_{m}=1.8 \mathrm{~mm}$ is the upper margin of study as for higher values of $A_{m}$, the structure will no longer be matched at the operating frequency. For $A_{m}>1.8 \mathrm{~mm}$, the travelling waves in Region 2 (see Fig. 3) face with an abrupt variation of lateral walls in Region 3 and the motion of waves would be affected so that the matching condition would no longer hold. However, it is expected that if the matching condition can be satisfied, the curves would follow similar pattern of movement for limited increment in $A_{m}$ value as shown in Fig. 6 for the case $w_{s}=0.3 \mathrm{~mm}$. The estimated data in this case are derived by curve-fitting technique, which expand the upper limit of $A_{m}$ from $1.8 \mathrm{~mm}$ to $2.2 \mathrm{~mm}$. Further expansion of $A_{m}$ would break the unity of slot and the continuity of guided waves.

\section{Aperture Illumination Calculation to Control the SLL}

As can be observed from Fig. 5, the constructed radiation patterns have high SLL. In order to control the SLL, the distribution of $\alpha_{l}$ must be managed along the slot. In this design, a very low side lobe level of $-30 \mathrm{~dB}$ is set as the target. In practice, if a specific SLL is to be achieved, the design must be carried out for a somewhat lower SLL [28]. Thus, to realize the SLL $=-30 \mathrm{~dB}$, the structure should be

\footnotetext{
${ }^{1}$ Note that with the smooth variations of lateral walls (i.e. for relatively small values of $A_{m}$ ), Eq. (9) can also be applicable to calculate the effective axial wavenumber of $n=-1$ harmonic and consequently $\beta_{-1} / k_{0}$.
}

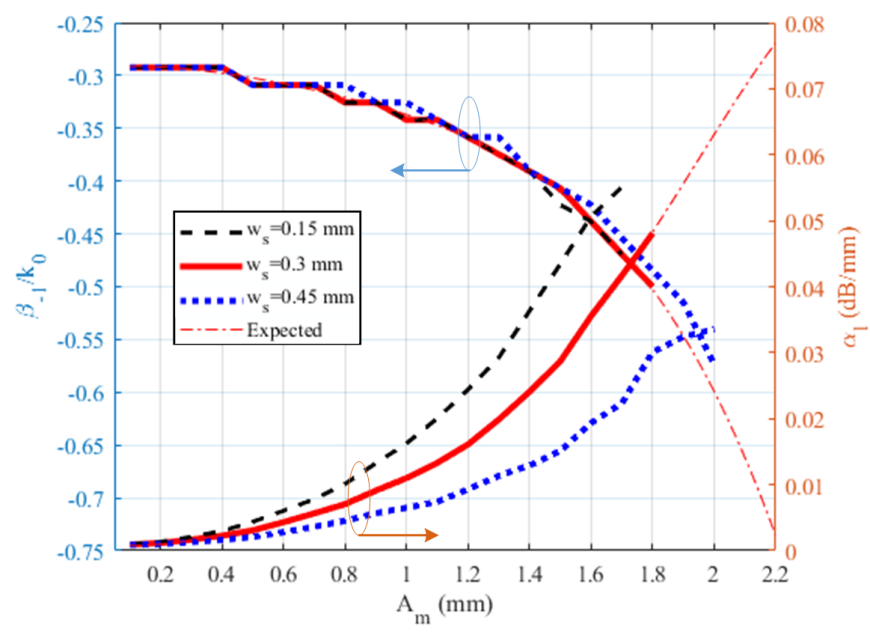

Fig. 6. The phase constant and leakage constant versus different values of $A_{m}$ for $N=23$.

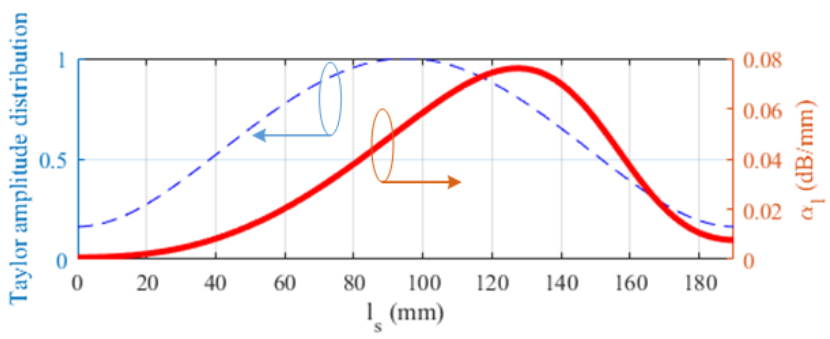

Fig. 7. Taylor aperture distribution for $-35 \mathrm{~dB}$ side-lobe levels and the corresponding leakage constant across the slot (the efficiency is set $90 \%$ ).

designed for side lobes of $35 \mathrm{~dB}$ below the main lobe. For SLL $>-20 \mathrm{~dB}$, overdesign would not normally be required.

Given an aperture distribution of $A(x)$ along the slot, the leakage distribution of $\alpha_{l}(x)$ can be calculated as follows;

$$
\alpha_{l}(x)=\frac{0.5|A(x)|^{2}}{\frac{1}{\eta_{a}} \int_{0}^{l_{s}}|A(\chi)|^{2} d \chi-\int_{0}^{x}|A(\chi)|^{2} d \chi},
$$

where $\eta_{a}$ is the radiation efficiency regarding to the antenna's radiating length $\left(l_{s}\right)$, defined as the ratio of radiated power to the injected one and is preferred to be within the range of $90 \%$ to $95 \%$ [1]. Considering $\eta_{a}=90 \%, A(x)$ and $\alpha_{l}(x)$ for the discussed design are presented in Fig. 7. With this calculated $\alpha_{l}$, the required $A_{m}$ distribution across the slot can be derived from Fig. 6 for the case $w_{s}=0.3 \mathrm{~mm}$.

According to Fig. 7, the required $\alpha_{l}$ is above $0.05 \mathrm{~dB} / \mathrm{mm}$ for $l_{s} \approx 90 \sim 150 \mathrm{~mm}$; mapping this specific region to Fig. 6, the corresponding $A_{m}$ must be calculated based on the "Expected" part of the curve.

\section{E. Deriving the Modulation Function}

If we have an invariant $\beta$ for all values of $\alpha_{l}$ in our structure, then $N=23$ with the modulated amplitude of $A_{m}$ (as explained in Section IV-D) could have resulted in the preset radiation characteristics, i.e. $\theta_{m}=-20 \mathrm{deg}$ and $\mathrm{SLL}=-30 \mathrm{~dB}$. However, as shown in Fig. 6, this cannot be 


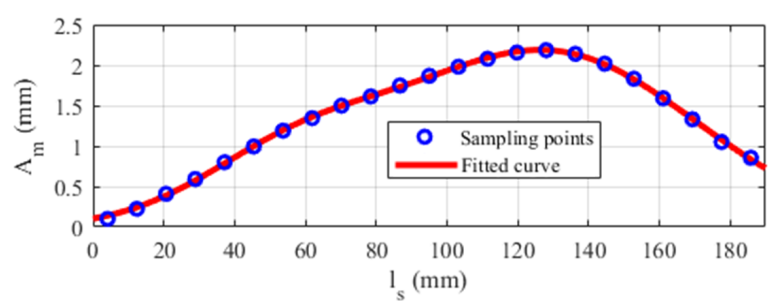

Fig. 8. Required $A_{m}$ distribution across the aperture considering the Figs. 6 and 7.

applicable: both $\beta$ and $\alpha_{l}$ vary as $A_{m}$ varies and this variation becomes severe for larger values of $A_{m}$.

Therefore, in this sub-section we aim to derive a modulation function $(\mathcal{M})$ to simultaneously describe the geometrical parameters of $N$ and $A_{m}$. With this function, both values of $\beta$ and $\alpha_{l}$ can be regulated at each individual point on $l_{s}$. The proposed method to extract the discussed function is broken into three steps as outlined below.

1) Analytic expression of the $A_{m}(x)$ : The slot's aperture is sampled by 23 equivalently-spaced points across $l_{s}$. With the aid of Fig. 7, each of these sample points can be mapped to a specific $\alpha_{l}$. Then, for all these 23 values of $\alpha_{l}$, corresponding values of $A_{m}$ are revealed by using Fig. 6. Finally, a leastsquares fitting is applied to the achieved data to derive $A_{m}(x)$ function:

$$
A_{m}(x)=2.161 e^{-\left(\frac{x-128.5}{58.87}\right)^{2}}+0.7718 e^{-\left(\frac{x-57.08}{38.44}\right)^{2}},
$$

where $x$ indicates the different locations on $l_{s}$.

The sampling points and the derived fitted curve (Eq. (12)) are shown in Fig. 8.

2) Analytic expression of the $N\left(A_{m}(x)\right)$ : Considering Figs. 5 (b) and 6, as $A_{m}$ varies, the tilt angle will also vary undesirably. This would cause different parts of the aperture to radiate toward different directions and would distort the constructed beam. To cope with this problem, as $A_{m}$ varies along the slot, the $S L$ must be varied purposefully to compensate the shift of $\beta_{-1} / k_{0}$.

Recalling $N=l_{s} / S L$, and following the same procedure as presented in the previous step for the curves of Figs. 5 (b) and $8, N\left(A_{m}(x)\right)$ is derived as follows;

$$
\begin{array}{r}
N\left(A_{m}(x)\right)=-2.218 \sin \left(A_{m}(x)-\pi\right) \\
+0.1923\left(A_{m}(x)-10\right)^{2}+5.576 .
\end{array}
$$

The sampling points and fitted curve are shown in Fig. 9 (a). By using Eq. (13), the corresponding pattern of $S L$ variation versus different values of $A_{m}$ and also versus the slot's length is calculated and presented in Fig. 9 (a) and (b) respectively.

3) Realizing the $\mathcal{M}$ function: The sinusoidal curve in Fig. 3 must be loyal to both required $A_{m}$ and $S L$ distributions that are presented in Figs. 8 and 9 (b). Note that defining $S L$ versus $l_{s}$ may sound unusual as the former is a function of a variable which is indeed making it. In other words, the dependency of $S L$ to $l_{s}$ is not only confined to the curve presented
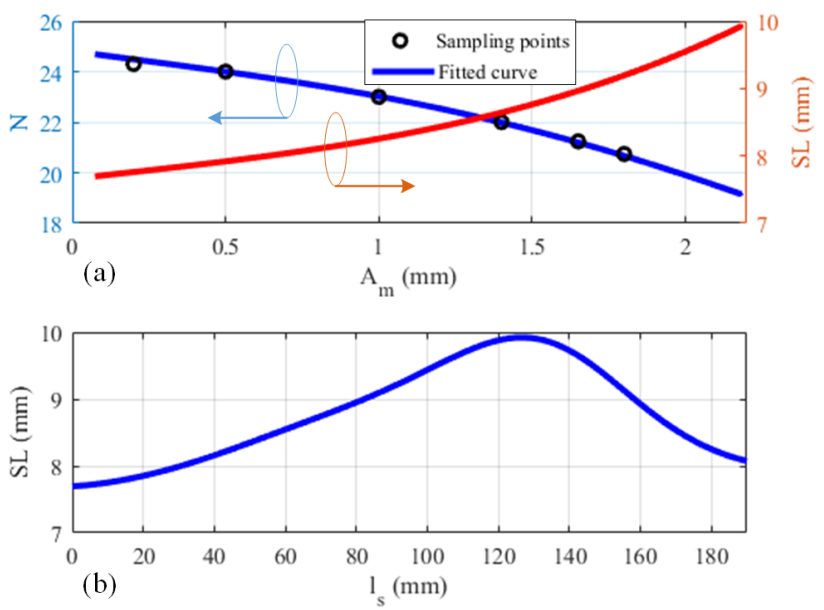

Fig. 9. (a) the sampling points and fitted curve of $N\left(A_{m}(x)\right)$ and the corresponding $S L$, (b) the required $S L(x)$ distribution across the aperture.

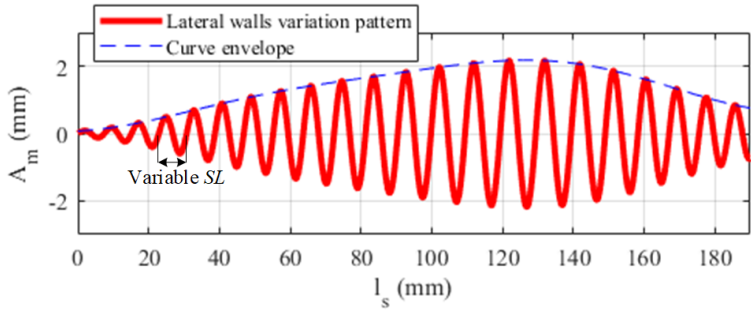

Fig. 10. The required sinusoidal pattern of lateral walls in Region 3 of the proposed ESIW (see Fig. 3).

in Fig. 9 (b), but also to the fact that $\sum S L=l_{s}$. This contradiction originates from the innate behavior of structure: $A_{m}$ is a function of length across the aperture, at the same time $S L$ (or $N$ ) is dependent on $A_{m}$ which makes the $S L$ a function of length as well.

In order to overcome the aforementioned issues, a proper modulation function must be defined to force both $A_{m}$ and $N$ to follow the required curve-patterns at each local point on the aperture continuously and simultaneously. Our proposed function is as follows:

$$
\begin{aligned}
\mathcal{M}= & A_{m}(x) \sin \left(\frac{2 \pi N_{m}}{l_{s}}\left(1-s_{\Delta}\right) x\right. \\
& \left.+2 \pi s_{\Delta} \int^{x} \frac{N\left(A_{m}(x)\right)}{l_{s}} d x\right),
\end{aligned}
$$

where $A_{m}(x)$ and $N\left(A_{m}(x)\right)$ are specified by Eqs. (12) and (13) respectively, $N_{m}$ is the mean value of $N$ across the aperture, and $s_{\Delta}$ represents the maximum $S L$ shift with respect to $S L_{m}=l_{s} / N_{m}$.

Considering Fig. 9 (a), $N_{m}=21.578$ and $s_{\Delta}=1.115$; then by employing the Eq. (14), the required sinusoidal pattern of the lateral walls is derived as presented in Fig. 10. 


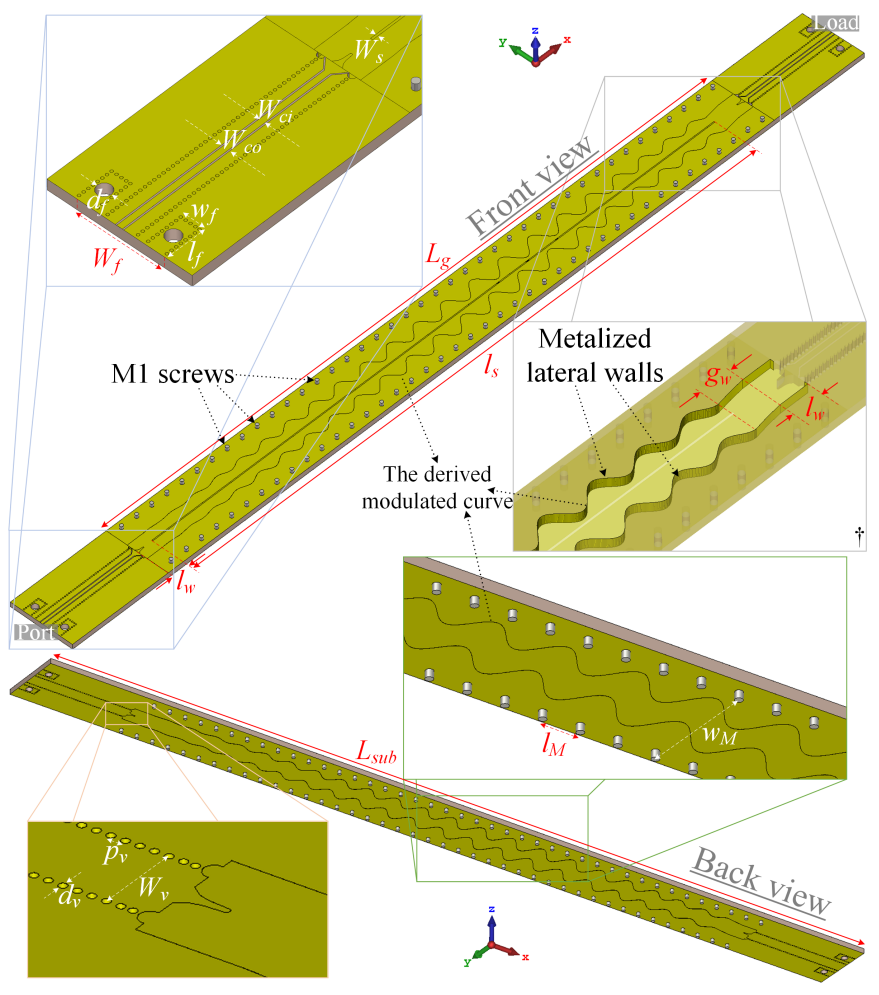

Fig. 11. The proposed long slot LWA with the designed SMGS based on ESIW technology.

\section{F. Applying the Modulation Function on the Geometry of Structure}

The proposed long slot LWA with the designed SMGS based on ESIW technology is shown in Fig. 11 from different angles/scales of observation. The geometrical parameters are $W_{f}=12.11 \mathrm{~mm}, d_{f}=1.98 \mathrm{~mm}, l_{f}=4.69 \mathrm{~mm}$, $w_{f}=2.58 \mathrm{~mm}, W_{c o}=1.183 \mathrm{~mm}, W_{c i}=0.783 \mathrm{~mm}$, $W_{s}=0.3 \mathrm{~mm}, L_{g}=205 \mathrm{~mm}, l_{s}=190 \mathrm{~mm}, l_{w}=5 \mathrm{~mm}$, $g_{w}=5 \mathrm{~mm}, w_{M}=16 \mathrm{~mm}, l_{M}=5 \mathrm{~mm}, L_{s u b}=267.84 \mathrm{~mm}$, $W_{v}=4.183 \mathrm{~mm}, p_{v}=0.67 \mathrm{~mm}$, and $d_{v}=0.4 \mathrm{~mm}$. Other geometrical parameters that are not depicted in this figure are equivalent to their counterparts in Fig. 1 with an obvious exception of $o_{s}=0 \mathrm{~mm}$ as the slot is now located in the middle.

The two thin lower and upper metallic sheets (see Fig. 1) are now attached to the substrate via M1 screws that are uniformly separated from each other. The transmission line at Region 1 (see Fig. 3) of the structure is selected to be the grounded coplanar waveguide (GCPW) instead of a conventional microstrip. The GCPW can make the traveling waves highly confined across the line, as well as inside the substrate. This can decrease the fringing fields of the line, making the leakage primarily loyal to the designed aperture.

The modulated lateral walls capture their patterns from Fig. 10. The calculated curve of Fig. 10 has $A_{m}=0 \mathrm{~mm}$ at $l_{s}=0 \mathrm{~mm}$ and reaches $\left|A_{m}\right| \approx 0.75 \mathrm{~mm}$ at $l_{s}=190 \mathrm{~mm}$. As a result, the physical shape of the consequent SMGS would not reach the same points at both ends of the slot which could break the symmetry of the structure to a certain extend. To tackle this issue, a region with the length of $g_{w}$ is added when

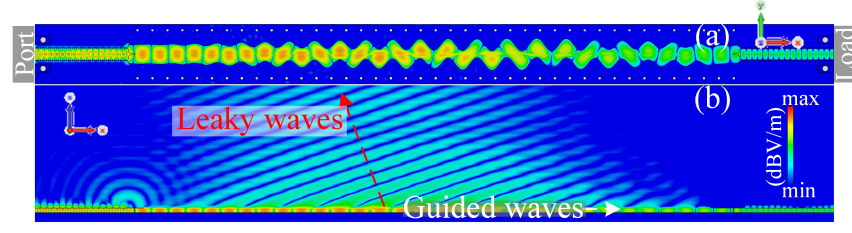

Fig. 12. |E-field $\mid$ distribution pattern at (a) top and (b) side view of the proposed long slot LWA at $f=35 \mathrm{GHz}$. The mechanism of leakage can be observed from the side-view plot.

the slot ends to make the load port be aligned with the exciting port (see Fig. $11 \dagger$ ).

It should be noted that some other methods have been presented in the literature to taper two physical parameters in an LWA [29]-[32]. However, to the best of our knowledge there is no modulation method like we proposed for manipulating such a relatively wide variation range of $\beta_{-1} / k_{0}$ and $\alpha_{l}$ (see Fig. 6) in an LWA.

\section{Simulation AND EXPERIMENT}

In this section, we describe the performance of the designed structure. Simulation and measurement results are presented to prove the practicality of our proposed method to control both tilt angle and SLL with a low cross polarization level.

\section{A. E-field Distribution, Conduction Loss, and Leakage Rate}

Recall that the proposed structure aims to achieve an inclined backward beam toward $\theta_{m}=-20$ deg with SLL $=$ $-30 \mathrm{~dB}$ at $f=35 \mathrm{GHz}$. The CST-MWS is used to simulate the designed LWA. Fig. 12 presents the E-field distribution associated with the antenna in two cutting plans of (a) top and (b) side view (across the middle of substrate). Considering Fig. 12 (a), the gradual loss of E-fields inside the SMGS is observable. This loss (i.e. $\alpha_{l}+\alpha_{c}$ ) is dominantly originated from the controlled leakage of wave throughout the slot as shown in Fig. 12 (b) which also displays that the nature of leakage is in the backward direction to the angle of interest.

To gain a clear understanding on the values of $\alpha_{c}$ and $\alpha_{l}$ in the designed SMGS, a series of specific simulations must be carried out in the CST-MWS. Let us start with the calculation of $\alpha_{c}$. First, we set the conducting wall's material as copper in the simulation setup as it is meant to be in the final prototype. Two E-field probes are then defined inside the air-filled guide at $\left(x=\left\{0, l_{s}\right\}, y=0, z=\frac{h}{2}\right)$ as shown in Fig. 13 (a). The absolute values of $\vec{E}$ at the corresponding probes are then recorded. Note that $|\vec{E}|_{\# 2}=|\vec{E}|_{\# 1} e^{-\left(\alpha_{l}+\alpha_{c}\right) l_{s}}$ which makes it possible to extract the total loss of $\alpha_{t 1}=\alpha_{l}+\alpha_{c}$ by comparing $|\vec{E}|_{\# 1}$ and $|\vec{E}|_{\# 2}$. Afterward, the material of the conducting walls are changed in the simulation setup to the perfect-electric-conductor (PEC) and the same procedure is repeated to record the modified values of $|\vec{E}|_{\# 1}$ and $|\vec{E}|_{\# 2}$. This time, $|\vec{E}|_{\# 2}=|\vec{E}|_{\# 1} e^{-\alpha_{l} l_{s}}$ as $\alpha_{c}=0$, which results in $\alpha_{t 2}=\alpha_{l}$. Finally, $\alpha_{c}=\alpha_{t 1}-\alpha_{t 2}$ (in $\left.\mathrm{dB} / \mathrm{mm}\right)$ can be derived for the designed SMGS. To examine the accuracy of the proposed method, it can be applied to an analogous straight ESIW. This provides us the opportunity to compare the 

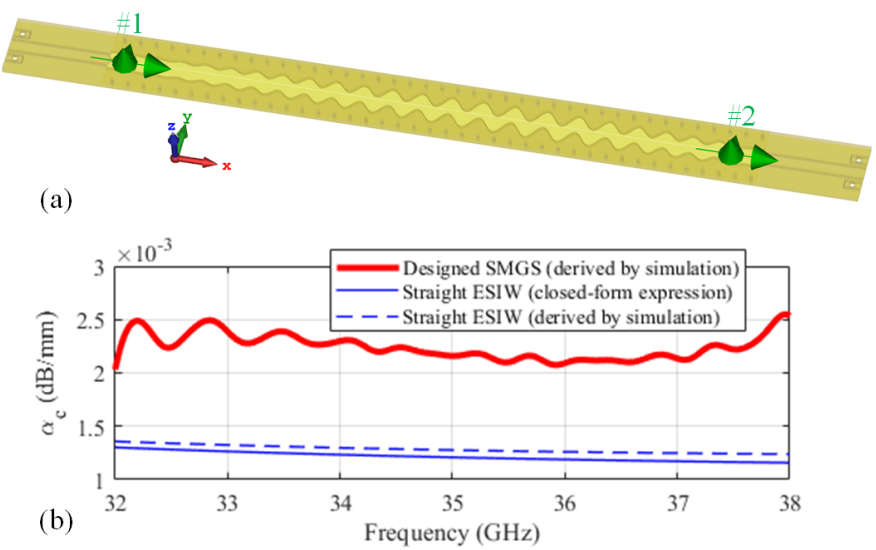

Fig. 13. (a) the simulation setup with copper as the conducting walls; the same simulation must be repeated by replacing copper with PEC. (b) the derived conduction loss across the designed SMGS. The conduction loss of the corresponding straight ESIW is also reported for comparison purposes only.

derived simulation-based $\alpha_{c}$ with the closed-form expression of Eq. (4). The results are plotted in Fig. 13 (b), showing the values of $\alpha_{c}$ at different frequencies and clarifying the practicality of the proposed method. It can be observed from this figure that forming a straight ESIW to the proposed SMGS increases the average value of $\alpha_{c}$ by almost $0.001 \mathrm{~dB} / \mathrm{mm}$.

We now aim to calculate the $\alpha_{l}$ across the slot of the designed structure to check if it follows the preset desired Taylor-based distribution of Fig. 7. The corresponding simulation setup is presented in Fig. 14 (a). There are twenty E-field probes at $\left(x=\left\{0, \Delta l, 2 \Delta l, \ldots, l_{s}\right\}, y=0, z=\frac{h}{2}\right)$ with $\Delta l=10 \mathrm{~mm}$ to sample the guided waves across the SMGS every $10 \mathrm{~mm}$. The conducting walls are PEC to eliminate the effect of $\alpha_{c}$ so that $|\vec{E}|_{\# g}=|\vec{E}|_{\# g-1} e^{-\alpha_{l} \Delta l}$ with $g=\{2,3, . ., 20\}$. Therefore, with a known couple of $\left(|\vec{E}|_{\# g},|\vec{E}|_{\# g-1}\right)$, it is possible to derive the value of $\alpha_{l}$ at the corresponding sampling point. The obtained values of $|\vec{E}|$ and $\alpha_{l}$ at $f=35 \mathrm{GHz}$ are shown in Fig. 14 (b). The $\alpha_{l}$ distribution of Fig. 7 is also replotted here (the closedform expression) for comparison purposes only. The presented results show that the designed SMGS generates an $\alpha_{l}$ which is reasonably matched with the preset distribution of Eq. (11). It is also worth mentioning that based on the ideal closedform expression of $\alpha_{l}$ (Eq. (11) and Fig. 7), the total leakage rate is $\int_{0}^{l_{s}} \alpha_{l} d l_{s}=6.36 \mathrm{~dB} / l_{s}$ in theory. Concerning Fig. 14, $|\vec{E}|_{\# 1}-|\vec{E}|_{\# 20}=\alpha_{l} l_{s}=6.57 \mathrm{~dB}$ which is very close to the theoretical value.

\section{B. Prototyping}

The structure is fabricated as presented in Fig. 15 from different angles; the substrate with the routed designed guide, the two copper sheets, and the assembled structure are shown in part (a) of this figure. The copper sheets should be thin to provide a proper leakage mechanism from the slot on top. But at the same time, they must be physically robust whereas an extremely thin copper sheet might be deformed easily with a subtle external pressure; more importantly, the

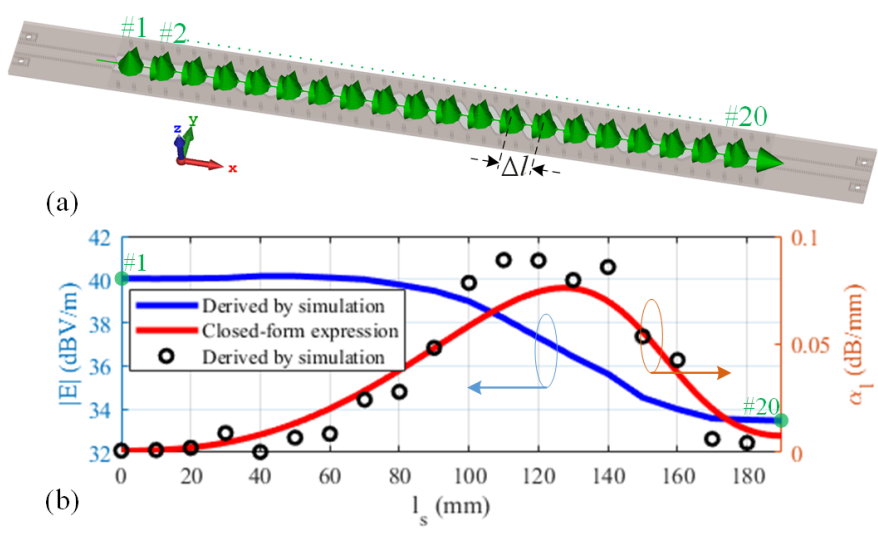

Fig. 14. (a) the simulation setup with PEC as the conducting walls. (b) the absolute electric field and $\alpha_{l}$ across the slot of the designed SMGS.

straightness of the long slot would be jeopardized in this case. Taking the above mentioned criterion into account, the thickness of the copper sheet is selected to be $0.2 \mathrm{~mm}$. The processing of the copper sheets was completed by stacking them between melamine material prior to machining. The slot was machined by incrementally stepping down into the copper using cutting fluid. The substrate processing was performed as a conventional PCB forming.

Fig. 15 (b), (c), and (d) show the magnified views of the transition region at both ends and the lateral metallized walls respectively. The top copper sheet is attached to the substrate via M1 screws (Fig. 15 (c, e)) and then the back copper sheet is added to the structure in hand to make the final assembled prototype (Fig. 15 (f)). Two $2.92 \mathrm{~mm}$ jack end-launch connectors are attached to both ends as shown in Fig. 15 (f-1, 4). A portion of structure is presented in Fig. 15 (f-3) from the side-view. The connectors not only cast as the EM-source/ load, but also assist in bonding the back copper sheet to the substrate more tightly (see Fig. 15 (f-2, 5)).

\section{S-parameter, Efficiency, and Radiation Pattern}

The scattering parameters of the designed LWA are plotted in Fig. 16 (a), specifying that the measurement results are well matched with the CST-MWS simulations. The measured $\left|\mathrm{S}_{11}\right|$ is below $-10 \mathrm{~dB}$ in the frequency range of $30.8 \mathrm{GHz}$ to $39.45 \mathrm{GHz}$.

Considering Eq. (10), the percentage of power radiated is $100(1-P(L) / P(0))$ so that the efficiency can be theoretically derived from the S-parameters as a good approximation. This theoretical $\eta_{a}$ is calculated from the simulated and measured S-parameters as presented in Fig. 16 (b), confirming that $\eta_{a} \approx$ $90 \%$ over the operating frequencies which is in agreement with the initial target set in Section IV-D. The simulated and measured $\eta_{a}$ and the total efficiency $\left(\eta_{t}\right)$ are also shown in this figure. Note that the reported efficiencies are measured based on the Directivity/Gain method presented in [33].

With known values of $S_{11}$ and $S_{21}$, the other important parameter that can be examined by Eq. (10) is $\alpha$. Consequently, $\alpha=7.59 \mathrm{~dB} / L_{\text {sub }}$ is derived from the simulated S-parameters 

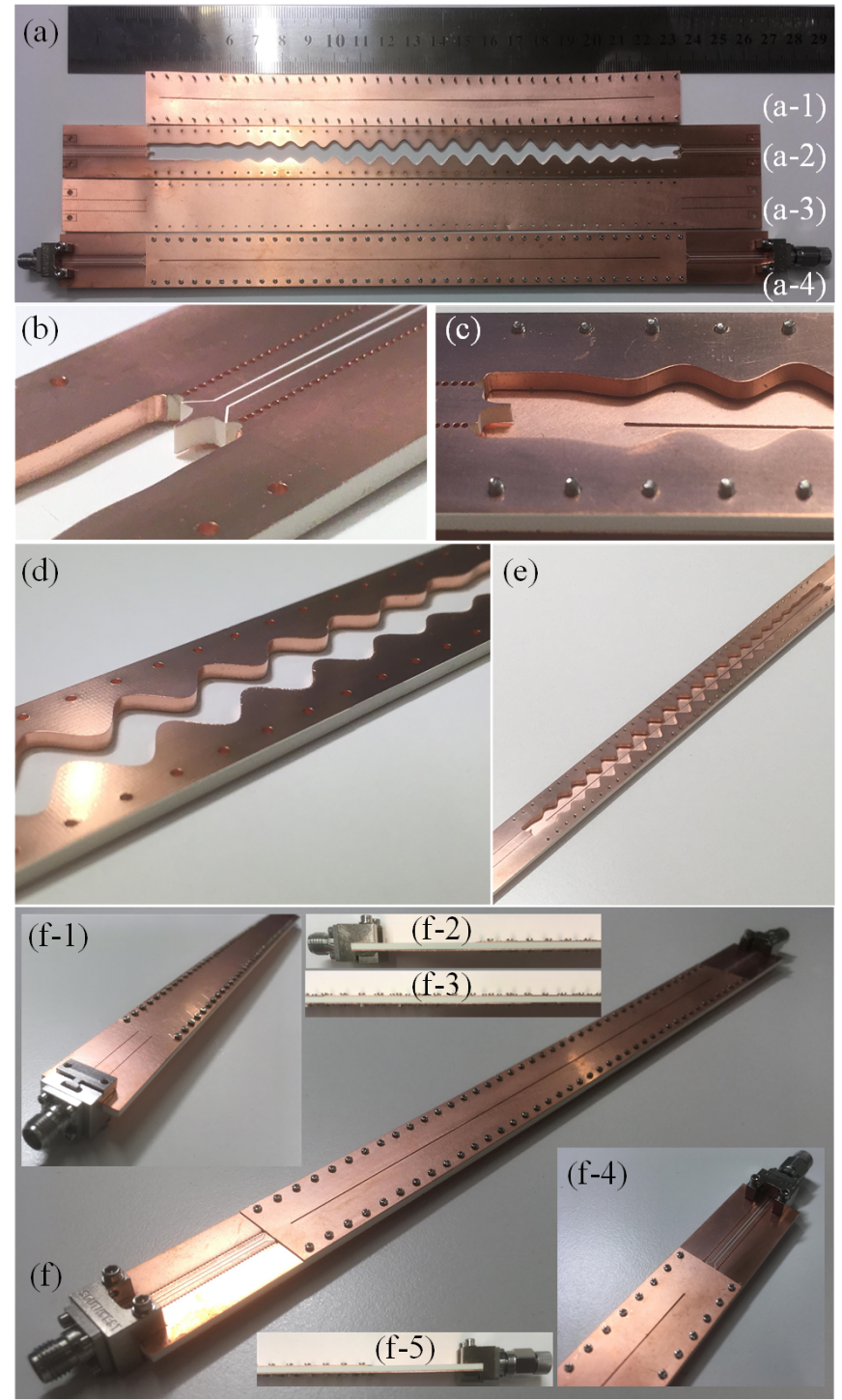

Fig. 15. The proposed long-slot SMGS-LWA on ESIW. (a) all building blocks: (a-1) the top slotted copper sheet, (a-2) the substrate with the routed guide, (a3) the back copper sheet, (a-4) the assembled structure. (b), (c) the transition from the microstrip line to the guide from the top, and back side angle of view respectively. (d), (e) the SMGS from the top, and back side angle of view respectively. (f) the assembled structure: (f-1) port end backside, (f-2, $3)$ side view at the port end and in the middle of structure, $(f-4,5)$ load end top, and side view respectively.

at $f=35 \mathrm{GHz}$, indicating the total end-to-end loss across the structure. Considering Fig. 13 (b), the overall conduction loss is $\alpha_{c}=0.42 \mathrm{~dB} / l_{s}$ at $f=35 \mathrm{GHz}$. Additionally, it is stated in Section V-A that the structure produces a leakage rate of $\alpha_{l}=6.57 \mathrm{~dB} / l_{s}$. Hence, it can be deduced that the accumulative loss in the GCPW lines at both ends of the structure is $7.59-(6.57+0.42)=0.6 \mathrm{~dB}$. Note that $\alpha=9.61 \mathrm{~dB} / L_{\text {sub }}$ based on the measured S-parameters.

Recall that in Section IV-C, it is explained that the structure cannot be matched for $A_{m}>1.8 \mathrm{~mm}$ in an overall condition. But we expected to have a matched structure provided that the $A_{m}$ variation occurs gradually. Based on this premise, the behavior of $\alpha_{l}$ and $\beta_{-1} / k_{0}$ were then estimated as presented in Fig. 11. This figure was the base of all other calculations
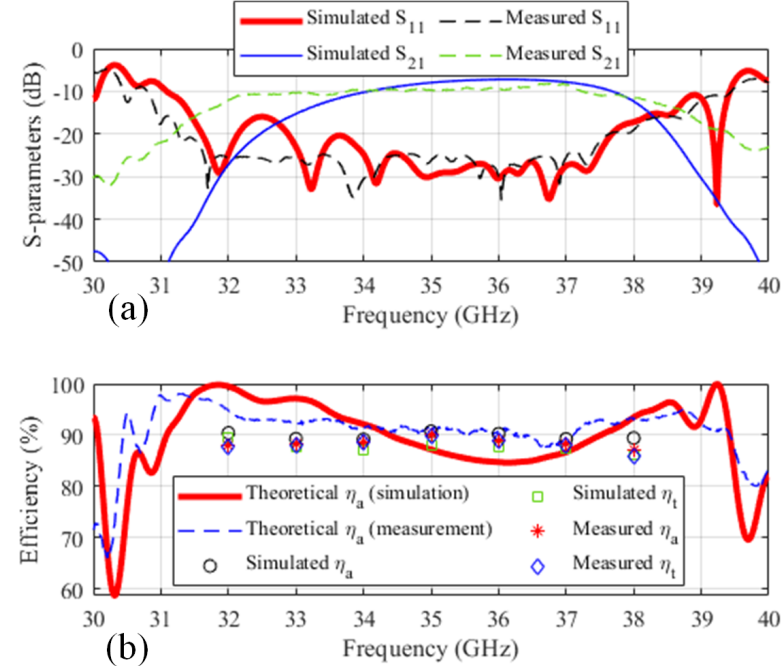

Fig. 16. (a) measured and simulated S-parameters, (b) the corresponding efficiencies.

in our proposed method. As discussed in Section IV-D, the SMGS must have $A_{m}>1.8 \mathrm{~mm}$ for $l_{s} \approx 90 \sim 150 \mathrm{~mm}$ which is also clarified in the derived curve of Fig. 10. Upon looking at the $\left|\mathrm{S}_{11}\right|$ results shown in Fig. 16 (a), it can be seen despite having $A_{m}>1.8 \mathrm{~mm}$ for the mentioned region of slot, the structure is matched well at the frequency of interest. This proves the validity of the method and verifies our conjecture. Moreover, the continuity of E-filed distribution in Fig. 12 is another consequence of the gradual $A_{m}$ variation which enables the aperture to be illuminated entirely and properly.

Fig. 17 (a) shows the normalized simulated and measured co-pol and cross-pol radiation patterns of the designed structure at $f=35 \mathrm{GHz}$ with the simulated (measured) realized gain of 18.81 (18.34) dBi. As can be observed from this plot, the constructed beam is well inclined to the angle of interest (e.g. $-20 \mathrm{deg}$ ) with simulated (measured) SLL $=-30.15$ $(-29.62) \mathrm{dB}$ and a very low cross-pol level. In order to study the frequency scanning capability of the designed LWA, the radiation characteristics are also assessed at $f=33 \mathrm{GHz}$ and $f=37 \mathrm{GHz}$ as illustrated in Fig. 17 (b) and (c) respectively. Fig. 17 (d) shows the simulated and measured realized gain over different frequencies. The measured tilt angle is also reported in this figure. It is observed that the tilt angle is moved by around $5 \mathrm{deg}$ on average at every $1 \mathrm{GHz}$ shift of frequency.

\section{CONCLUSIONS}

With an introduction on long slot LWA's design based on ESIW, a method is proposed to manipulate the electromagnetic fields on a periodic form of this antenna type. The lateral walls of the ESIW follow a sinusoidal curve which give their physical properties from a derived modulation function. With this method, it is possible to simultaneously control both $\beta$ and $\alpha$ in the guided-wave structure and, consequently, the tilt angle and SLL of the constructed beam. As the slot is placed on the centerline of the broad wall of the ESIW, the cross polarization is reduced properly. A simulation-based technique is proposed and applied to study the conduction loss of the designed guide, 

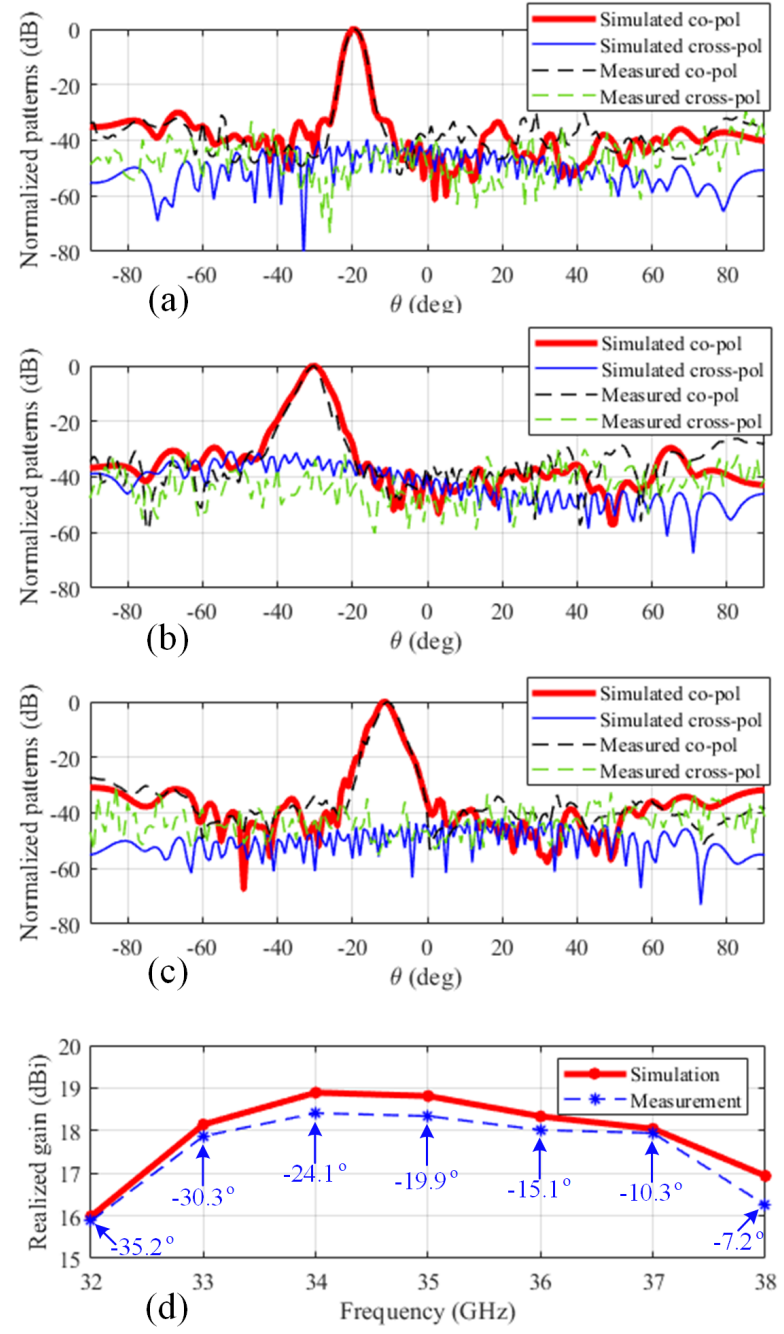

Fig. 17. Normalized radiation patterns for (a) $35 \mathrm{GHz}$ (the frequency of interest), (b) $33 \mathrm{GHz}$, and (c) $37 \mathrm{GHz}$. (d) the realized gain over different frequencies with the corresponding tilt angles.

as well as the leakage rate distribution across the radiating slot. The proposed structure is fabricated and measured, and the antenna performance are found to have an excellent agreement with what is eventuated theoretically and what is obtained in the simulations. The beam-scanning characteristic versus frequency, alongside the antenna's efficiency and realized gain are studied for the designed LWA.

\section{REFERENCES}

[1] A. Oliner and D. Jackson, "Antenna engineering handbook, j. volakis, ed," 2007.

[2] F. Monticone and A. Alu, "Leaky-wave theory, techniques, and applications: from microwaves to visible frequencies," Proceedings of the IEEE, vol. 103, no. 5, pp. 793-821, 2015.

[3] F. Xu and K. Wu, "Understanding leaky-wave structures: A special form of guided-wave structure," IEEE Microwave Magazine, vol. 14, no. 5, pp. 87-96, 2013.

[4] D. R. Jackson and A. A. Oliner, "Leaky-wave antennas," Modern antenna handbook, pp. 325-367, 2008.

[5] J. Liu, D. R. Jackson, and Y. Long, "Modal analysis of dielectricfilled rectangular waveguide with transverse slots," IEEE transactions on antennas and propagation, vol. 59, no. 9, pp. 3194-3203, 2011.
[6] G. Minatti, M. Faenzi, E. Martini, F. Caminita, P. De Vita, D. GonzálezOvejero, M. Sabbadini, and S. Maci, "Modulated metasurface antennas for space: Synthesis, analysis and realizations," IEEE Transactions on Antennas and Propagation, vol. 63, no. 4, pp. 1288-1300, 2014.

[7] F. L. Whetten and C. A. Balanis, "Meandering long slot leaky-wave waveguide-antennas," IEEE Transactions on antennas and propagation, vol. 39, no. 11, pp. 1553-1560, 1991.

[8] Y. J. Cheng, W. Hong, K. Wu, and Y. Fan, "Millimeter-wave substrate integrated waveguide long slot leaky-wave antennas and twodimensional multibeam applications," IEEE Transactions on Antennas and Propagation, vol. 59, no. 1, pp. 40-47, 2010.

[9] D. Deslandes and K. Wu, "Accurate modeling, wave mechanisms, and design considerations of a substrate integrated waveguide," IEEE Transactions on microwave theory and techniques, vol. 54, no. 6, pp. 2516-2526, 2006.

[10] T. Djerafi, A. Doghri, and K. Wu, "Substrate integrated waveguide antennas," Handbook of Antenna Technologies, pp. 1585-1655, 2016.

[11] D. Zheng, Y.-L. Lyu, and K. Wu, "Longitudinally slotted siw leaky-wave antenna for low cross-polarization millimeter-wave applications," IEEE Transactions on Antennas and Propagation, 2019.

[12] A. Mallahzadeh and S. Mohammad-Ali-Nezhad, "Long slot ridged siw leaky wave antenna design using transverse equivalent technique," IEEE Transactions on Antennas and Propagation, vol. 62, no. 11, pp. 54455452,2014

[13] S. Mohammad-Ali-Nezhad and A. Mallahzadeh, "Periodic ridged leakywave antenna design based on siw technology," IEEE Antennas and Wireless Propagation Letters, vol. 14, pp. 354-357, 2015.

[14] A. R. Mallahzadeh and M. H. Amini, "Design of a leaky-wave long slot antenna using ridge waveguide," IET Microwaves, Antennas \& Propagation, vol. 8, no. 10, pp. 714-718, 2014.

[15] A. Belenguer, H. Esteban, and V. E. Boria, "Novel empty substrate integrated waveguide for high-performance microwave integrated circuits," IEEE transactions on microwave theory and techniques, vol. 62 , no. 4 , pp. 832-839, 2014.

[16] H. Esteban, A. Belenguer, J. R. Sánchez, C. Bachiller, and V. E. Boria, "Improved low reflection transition from microstrip line to empty substrate-integrated waveguide," IEEE Microwave and Wireless Components Letters, vol. 27, no. 8, pp. 685-687, 2017.

[17] A. Belenguer, H. Esteban, A. L. Borja, and V. E. Boria, "Empty siw technologies: A major step toward realizing low-cost and low-loss microwave circuits," IEEE Microwave Magazine, vol. 20, no. 3, pp. 24-45, 2019.

[18] J. Mateo, A. M. Torres, A. Belenguer, and A. L. Borja, "Highly efficient and well-matched empty substrate integrated waveguide h-plane horn antenna," IEEE Antennas and Wireless Propagation Letters, vol. 15, pp. 1510-1513, 2016.

[19] Z. U. Khan, A. Alomainy, and T. H. Loh, "Empty substrate integrated waveguide planar slot antenna array for 5g wireless systems," in 2019 IEEE International Symposium on Antennas and Propagation and USNC-URSI Radio Science Meeting. IEEE, 2019, pp. 1417-1418.

[20] Z. U. Khan, A. Belenguer, T. H. Loh, and A. Alomainy, "Experimental investigation of empty substrate integrated waveguide-fed $\mathrm{mmw}$ patch antenna for 5g applications," in 2019 UK/China Emerging Technologies (UCET). IEEE, 2019, pp. 1-3.

[21] Z. U. Khan, T. H. Loh, A. Belenguer, and A. Alomainy, "Empty substrate-integrated waveguide-fed patch antenna array for $5 \mathrm{~g}$ millimeter-wave communication systems," IEEE Antennas and Wireless Propagation Letters, vol. 19, no. 5, pp. 776-780, 2020.

[22] A. Hessel, "General characteristics of traveling-wave antennas, antenna theory-part 2, chapter 19, 1969."

[23] D. R. Jackson, C. Caloz, and T. Itoh, "Leaky-wave antennas," Proceedings of the IEEE, vol. 100, no. 7, pp. 2194-2206, 2012.

[24] A. Araghi, M. Khalily, P. Xiao, and R. Tafazolli, "Holographic-based leaky-wave structures: Transformation of guided waves to leaky waves," IEEE Microwave Magazine, vol. 22, no. 6, pp. 49-63, 2021.

[25] J. H. Choi and T. Itoh, "Beam-scanning leaky-wave antennas," in Handbook of Antenna Technologies, Z. N. Chen, D. Liu, H. Nakano, X. Qing, and T. Zwick, Eds. Singapore: Springer, 2016, pp. 16971735.

[26] RO4360G2 $2^{T M}$ High Frequency Laminates DATASHEET, Rogers corporation.

[27] C. A. Balanis, "Advanced engineering electromagnetics." John Wiley \& Sons, Inc, 2012, ch. 8, p. 377.

[28] R. E. Collin, "Antennas and radiowave propagation." McGraw-Hill, 1985, ch. 4, p. 268. 
[29] J. L. Gómez-Tornero, A. T. Martinez, D. C. Rebenaque, M. Gugliemi, and A. Álvarez-Melcón, "Design of tapered leaky-wave antennas in hybrid waveguide-planar technology for millimeter waveband applications," IEEE transactions on antennas and propagation, vol. 53, no. 8 , pp. 2563-2577, 2005.

[30] J. L. Gomez-Tornero, G. Goussetis, A. P. Feresidis, and A. A. Melcón, "Control of leaky-mode propagation and radiation properties in hybrid dielectric-waveguide printed-circuit technology: Experimental results," IEEE transactions on antennas and propagation, vol. 54, no. 11, pp. 3383-3390, 2006.

[31] A. J. Martinez-Ros, J. L. Gomez-Tornero, and G. Goussetis, "Planar leaky-wave antenna with flexible control of the complex propagation constant," IEEE Transactions on Antennas and Propagation, vol. 60, no. 3, pp. 1625-1630, 2011.

[32] A. J. Martinez-Ros, J. L. Gómez-Tornero, and G. Goussetis, "Holographic pattern synthesis with modulated substrate integrated waveguide line-source leaky-wave antennas," IEEE Transactions on Antennas and Propagation, vol. 61, no. 7, pp. 3466-3474, 2013.

[33] Y. Huang, "Radiation efficiency measurements of small antennas," in Handbook of Antenna Technologies, Z. N. Chen, D. Liu, H. Nakano, X. Qing, and T. Zwick, Eds. Singapore: Springer, 2016, pp. 21652189. 